\title{
Two-Channel Metal Detector Using Two Perpendicular Antennas
}

\author{
Kyoo Nam Choi \\ Department of Information and Telecommunication Engineering, Incheon National University, Incheon 406-772, Republic of Korea \\ Correspondence should be addressed to Kyoo Nam Choi; knchoi@incheon.ac.kr
}

Received 6 March 2014; Revised 14 June 2014; Accepted 23 June 2014; Published 10 July 2014

Academic Editor: Ignacio R. Matias

Copyright (C) 2014 Kyoo Nam Choi. This is an open access article distributed under the Creative Commons Attribution License, which permits unrestricted use, distribution, and reproduction in any medium, provided the original work is properly cited.

\begin{abstract}
Two-channel metal detector, having two sets of perpendicularly oriented sensor antennas, is proposed to expand detectable size, ranging from $\mathrm{mm}$ through $\mathrm{cm}$ scale, of metal sensor, while conventional metal sensor is dedicated for detection only in mm or $\mathrm{cm}$ scale. The characteristics of the two metal detection sensor channels were investigated, respectively, and the interference effect, while in simultaneous operation, between two sensor channels was discussed. Metal detection channel, having sensitivity in mm scale, showed detectable sensitivity to moving ferrous sphere, with diameter down to $0.7 \mathrm{~mm}$, at $50 \mathrm{kHz}$ exciting frequency and enhanced sensitivity distribution. And metal detection channel having sensitivity in $\mathrm{cm}$ scale showed more uniform sensitivity distribution with the flexibility for future modular construction. The effect of interference, while in simultaneous operation of two sensors, resulted in reduced output response, but still within usable detection range. Thus it was feasible to operate two sensors, having different sensitivity range, simultaneously and to extend detection range from $\mathrm{mm}$ to $\mathrm{cm}$ scale, within practically acceptable interference.
\end{abstract}

\section{Introduction}

The metal detection sensor is now widely used in not only food processing industry but also defense industry $[1,2]$ to detect metal pieces in surrounding object, and considerable efforts have been devoted to enhance sensitivity and selectivity. Foreign materials are not only metal but also wood, ceramics [3], and germs [4], which are not detectable by using metal sensor. Other methods are envisaged to detect these materials which are not detectable by using metal sensor. Among these foreign materials, the detection for metal pieces is important [5] in food industry, and various researches are concentrating on this. The demand for metal detection is high in food industry, and recently the detection methods by using X-ray [6] and light [7] have been already applied for this. However the method using electromagnetic wave is dominant, and recently the method using superconducting coil has been attempted $[8,9]$. Theoretical analysis $[10,11]$ and sensitivity analysis $[12,13]$ along with the shape of metal sensor have been performed to explore the sensitivity of electromagnetic metal sensor. On the other hand, there were attempts [14] to enhance visibility of metal piece through signal processing. The parent body containing metal piece is important from the point of selectivity, and the effort to detect foreign pieces in powder has been attempted [15]. The structure of sensor head in metal detector plays an important role in terms of sensitivity, and relevant studies [16] have been conducted in this regard. Active researches have been performed for the various kinds and shapes of metal in metal sensor head [17]. However the single channel metal detection sensor has not shown sensitivity resolution through wide range of metal sizes from $\mathrm{mm}$ to $\mathrm{cm}$ scale. Thus there was a need to cascade the sensors having different sensitivity resolutions. Metal detection sensor, using electromagnetic wave, usually is strongly influenced by nearby electromagnetic wave which is within several times of sensor width; thus it is not feasible to place a second sensor in the vicinity of the first sensor. This paper is concerned with the experimental development of two channel metal sensors by cascading two metal sensors having different sensitivity resolution with minimum interference with each other. Two-perpendicular-antennas model is introduced to 
minimize physical interference, and the optimal signal detection method is investigated to reject the interference between metal sensors having different sensitivity resolution.

\section{Two-Antenna Model}

2.1. Single Channel Model. The conventional metal detection sensor with sensitivity resolution in $\mathrm{mm}$ scale has the antenna set, one transmitting antenna and two receiving antennas which are connected in opposite polarity to cancel reception signal while in steady state. In case when an object containing a nonspherical metal piece is fed through hollow antenna cross-sectional space as shown in Figure 1, then the sensor shows good sensitivity only for one directional position, between metal piece and antenna, where the perturbation in electromagnetic flux linkages becomes maximum. In the single channel model, the moving object containing metal piece perturbs gradually from first electromagnetic flux linkages between TX antenna \#1 and RX antenna \#1 to second electromagnetic flux linkages between TX antenna $\# 1$ and RX antenna \#2. The amounts of these spatially varying electromagnetic flux linkages induce currents into $\mathrm{RX}$ antennas \#1 and \#2, and the current difference between RX antennas \#1 and \#2 becomes output current, which represent the electromagnetic flux unbalance of antenna set $\# 1$.

The equivalent circuit of an antenna set, one transmitting and two receiving antennas, is shown as in Figure 2. The instantaneous output voltage $v_{10}$, in no load condition, can be expressed as the difference of mutual inductance, $M_{1}-M_{2}$, between transmitting and respective receiving antenna as shown in (1), and is also proportional to exciting frequency as shown in (3). In this model, the distance between transmitting antenna and receiving antenna is closer than the distance to passing object containing metal piece; thus antenna inductances are larger than mutual inductance, $M_{11}$ and $M_{12}$. If transmitting antenna is excited using sinusoidal signal, $e_{1 \mathrm{~s}}=$ $e_{10} \sin (\omega t+\delta)$, then antenna current becomes as shown in (2). Consider

$$
\begin{gathered}
v_{10}=v_{11}-v_{12}=\left(M_{11}-M_{12}\right) \frac{d i_{p 1}}{d t}, \\
i_{p 1}=j \frac{\omega L_{p 1} e_{10}}{\sqrt{\left(\omega L_{p 1}\right)^{2}+R_{p 1}^{2}}} \sin (\omega t+\delta), \\
v_{10}=j \frac{\omega^{2} L_{p 1} e_{10}\left(M_{11}-M_{12}\right)}{\sqrt{\left(\omega L_{p 1}\right)^{2}+R_{p 1}^{2}}} \cos (\omega t+\delta) .
\end{gathered}
$$

When the phasor expression is used for the flux linkage $\psi$, then the phasor voltage $V$ due to flux linkage can be expressed as in (4) where $I$ denotes phasor current:

$$
V_{10}=j \omega \psi_{10}=j \omega\left(M_{11}-M_{12}\right) I_{p 1} .
$$

It is noted that mutual inductance variance plays a key role in determining sensitivity of metal detection sensor.
The mutual inductance is expressed as in (5), in which $k_{11}$ and $k_{12}$ represent coupling coefficient between transmitting and receiving antennas, respectively. And these coupling coefficients have the value $0 \leq k_{11}, k_{12} \leq 1$ :

$$
M_{11}=k_{11} \sqrt{L_{p 1} L_{s 1 / 2}}, \quad M_{12}=k_{12} \sqrt{L_{p 1} L_{s 1 / 2}} .
$$

In steady state of initial measurement, $M_{11}$ and $M_{12}$ are adjusted to $M_{11}=M_{12}$, and output voltage becomes $v_{10} \approx 0$. When $\omega$ is higher and $M_{11}-M_{12}$ are bigger for the same size of metal piece, then it is advantageous in terms of sensitivity.

2.2. Two-Channel Model. In the two antenna sets as shown in Figure 3, the moving object after passing antenna set \#1 perturbs gradually from third electromagnetic flux linkages between TX antenna \#2 and RX antenna \#4 to fourth electromagnetic flux linkages between TX antenna \#2 and RX antenna \#3. Also the amounts of spatially varying electromagnetic flux linkages induce currents into RX antennas \#4 and $\# 3$, and the current difference between RX antennas \#4 and $\# 3$ becomes output current of antenna set \#2.

It is difficult to calculate the variance of mutual inductance for the moving object; thus experimental method is attempted. By using the model in Figure 4, the output voltage in each antenna set becomes the product of mutual inductance matrix and instantaneous current of each transmitting antenna as in

$$
\left[\begin{array}{l}
V_{10} \\
V_{20}
\end{array}\right]=j \omega\left[\begin{array}{ll}
M_{11}-M_{12} & M_{21}-M_{22} \\
M_{13}-M_{14} & M_{23}-M_{24}
\end{array}\right]\left[\begin{array}{c}
I_{p 1} \\
I_{p 2}
\end{array}\right]
$$

Assume $I_{T 11}$ is phasor current in one conductor of TX antenna $\# 1, D$ is the distance, $P$ in subscript is the remote point outside antenna, $T$ in first subscript alphabet is transmitting antenna, $R$ in first subscript alphabet is receiving antenna, the number in second subscript numeric is antenna number, the number in third subscript numeric is conductor number of antenna, and 4th through 6th alphabet and numeric follow the same convention as in 1st through $3 \mathrm{rd}$ alphabet and numeric. Then $D_{R 11 T 11}$ is the distance between one conductor of RX antenna \#1 and one conductor of TX antenna $\# 1$, and $D_{T 11 P}$ is the distance between one conductor of TX antenna $\# 1$ and a remote point $P$. The flux linkage $\psi_{R 11 T 11}$ with one conductor of RX antenna \#1 due to one conductor of TX antenna \#1 can be expressed as in

$$
\psi_{R 11 T 11}=2 \times 10^{-7}\left(I_{T 11} \ln \frac{D_{T 11 P}}{D_{R 11 T 11}}\right) .
$$




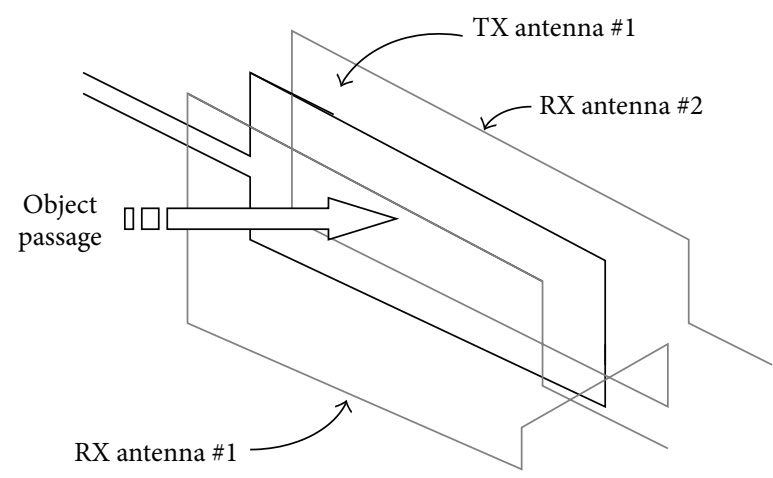

Figure 1: Conventional antenna set of metal detection sensor.

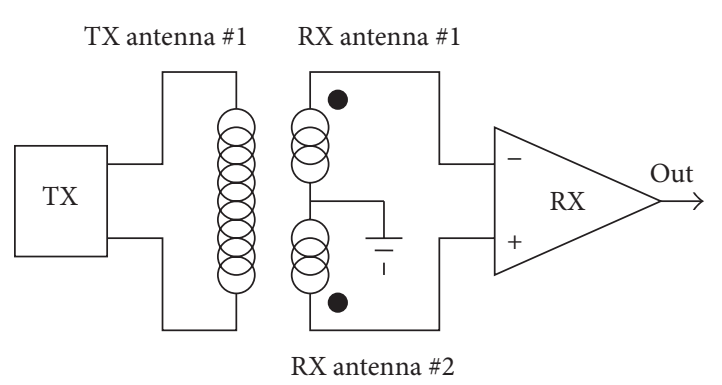

(a)

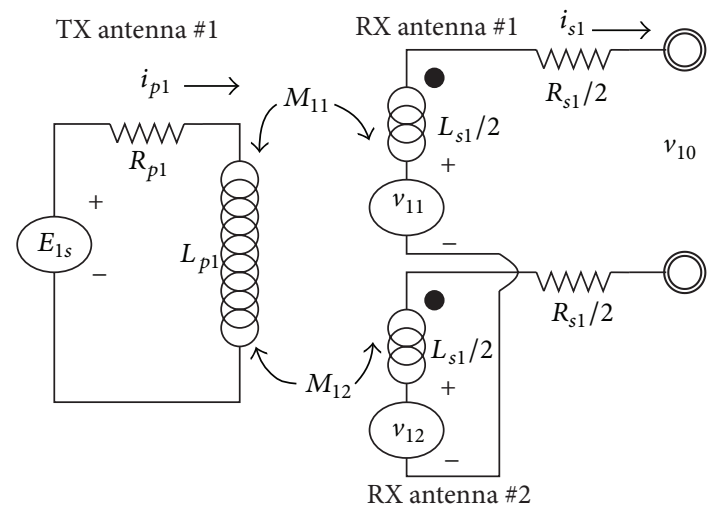

(b)

FIGURE 2: Equivalent circuit of antenna set: (a) schematic diagram and (b) equivalent circuit.

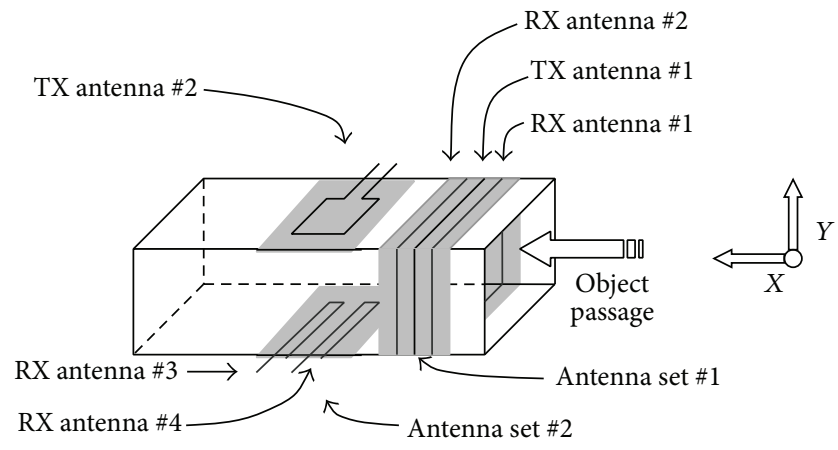

FIgURE 3: Two antenna sets of metal detection sensor.

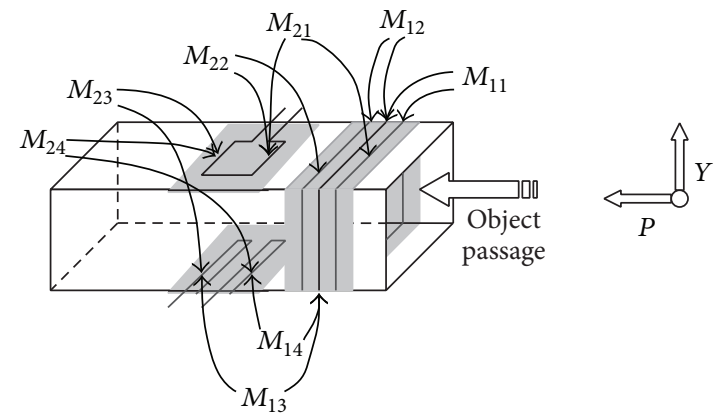

Figure 4: Sensor layout for two antenna sets. 
If we consider flux linkage to one conductor of receiving antenna \#1 from two transmitter antennas, this flux linkage $\psi_{R 11}$ can be expressed as in

$$
\begin{aligned}
& \psi_{R 11}=\psi_{R 11 T 11}+\psi_{R 11 T 12}+\psi_{R 11 T 21}+\psi_{R 11 T 22} \\
& =2 \times 10^{-7}\left(I_{T 11} \ln \frac{D_{T 11 P}}{D_{R 11 T 11}}+I_{T 12} \ln \frac{D_{T 12 P}}{D_{R 11 T 12}}\right. \\
& \left.+I_{T 21} \ln \frac{D_{T 21 P}}{D_{R 11 T 21}}+I_{T 22} \ln \frac{D_{T 22 P}}{D_{R 11 T 22}}\right) \\
& =2 \times 10^{-7}\left(I_{T 11} \ln \frac{1}{D_{R 11 T 11}}+I_{T 12} \ln \frac{1}{D_{R 11 T 12}}\right. \\
& +I_{T 21} \ln \frac{1}{D_{R 11 T 21}}+I_{T 22} \ln \frac{1}{D_{R 11 T 22}} \\
& +I_{T 11} \ln D_{T 11 P}+I_{T 12} \ln D_{T 12 P} \\
& \left.+I_{T 21} \ln D_{T 21 P}+I_{T 22} \ln D_{T 22 P}\right) \text {. }
\end{aligned}
$$

In this configuration, the sum of two currents in TX antenna \#1 is zero, $I_{T 11}+I_{T 12}=0$; also likewise $I_{T 21}+I_{T 22}=0$ for TX antenna \#2. Let the point $P$ move infinitely far away so that the set of terms containing logarithms of ratios of distances from $P$ becomes infinitesimal; then the ratio of the distances approaches 1 . Substituting these into (8) and recombining some logarithmic terms, we have (9) with the unit weber-turns/meter:

$$
\psi_{R 11}=2 \times 10^{-7}\left(I_{T 11} \ln \frac{D_{R 11 T 12}}{D_{R 11 T 11}}+I_{T 21} \ln \frac{D_{R 11 T 22}}{D_{R 11 T 21}}\right) .
$$

Thus flux linkage $\psi_{R 1}$ in RX antenna \#1 becomes sum of $\psi_{R 11}$ and $\psi_{R 12}$ as shown in

$$
\begin{aligned}
\psi_{R 1}=2 \times 10^{-7}( & I_{T 11} \ln \frac{D_{R 11 T 12}}{D_{R 11 T 11}}+I_{T 11} \ln \frac{D_{R 12 T 12}}{D_{R 12 T 11}} \\
& \left.+I_{T 21} \ln \frac{D_{R 11 T 22}}{D_{R 11 T 21}}+I_{T 21} \ln \frac{D_{R 12 T 22}}{D_{R 12 T 21}}\right) \\
=2 \times 10^{-7}( & \left(\ln \frac{D_{R 11 T 12}}{\left.D_{R 11 T 11}+\ln \frac{D_{R 12 T 12}}{D_{R 12 T 11}}\right) I_{T 11}}\right. \\
& \left.+\left(\ln \frac{D_{R 11 T 22}}{D_{R 11 T 21}}+\ln \frac{D_{R 12 T 22}}{D_{R 12 T 21}}\right) I_{T 21}\right) \\
=4 \times 10^{-7}( & \ln \frac{\sqrt{D_{R 11 T 12} D_{R 12 T 12}}}{\sqrt{D_{R 11 T 11} D_{R 12 T 11}}} I_{T 11} \\
& \left.+\ln \frac{\sqrt{D_{R 11 T 22} D_{R 12 T 22}}}{\sqrt{D_{R 11 T 21} D_{R 12 T 21}}} I_{T 21}\right) .
\end{aligned}
$$

Likewise all mutual inductances including $M_{11}$ and $M_{21}$ are expressed in a similar way as is shown in

$$
\begin{aligned}
& M_{11}=4 \times 10^{-7} \ln \frac{\sqrt{D_{R 11 T 12} D_{R 12 T 12}}}{\sqrt{D_{R 11 T 11} D_{R 12 T 11}}}, \\
& M_{21}=4 \times 10^{-7} \ln \frac{\sqrt{D_{R 11 T 22} D_{R 12 T 22}}}{\sqrt{D_{R 11 T 21} D_{R 12 T 21}}}, \\
& M_{12}=4 \times 10^{-7} \ln \frac{\sqrt{D_{R 21 T 12} D_{R 22 T 12}}}{\sqrt{D_{R 21 T 11} D_{R 22 T 11}}}, \\
& M_{22}=4 \times 10^{-7} \ln \frac{\sqrt{D_{R 21 T 22} D_{R 22 T 22}}}{\sqrt{D_{R 21 T 21} D_{R 22 T 21}}}, \\
& M_{13}=4 \times 10^{-7} \ln \frac{\sqrt{D_{R 31 T 12} D_{R 32 T 12}}}{\sqrt{D_{R 31 T 11} D_{R 32 T 11}}}, \\
& M_{23}=4 \times 10^{-7} \ln \frac{\sqrt{D_{R 31 T 22} D_{R 32 T 22}}}{\sqrt{D_{R 31 T 21} D_{R 32 T 21}}}, \\
& M_{24}=4 \times 10^{-7} \ln \frac{\sqrt{D_{R 41 T 22} D_{R 42 T 22}}}{\sqrt{D_{R 41 T 21} D_{R 42 T 21}}} . \\
& \sqrt{D_{R 41 T 12} D_{R 42 T 12}}
\end{aligned}
$$

When a foreign object, for example, a metal cube, passes through the above flux linkage, then the above mutual inductance $M$ will be perturbed, and the perturbed flux linkage is converted to voltage at sensor output port. For the metal sphere, having radius $a$, which is $x$ meters from the center of antenna conductor, the field intensity becomes $H_{x}=I / 2 \pi x$, and accordingly the flux density at the distance $x$ is $B_{x}=\mu I / 2 \pi x$ webers $/ \mathrm{m}^{2}$. Thus flux linkage by metal sphere becomes as in (12), and this amount perturbs the mutual inductances in steady state:

$$
\psi_{x}=\sum_{i=1}^{4} \frac{4 \pi a^{2} \mu I_{i}}{2 \pi x_{i}} .
$$

\section{Signal Detection Method}

3.1. Noise Rejection Using BPF. Two-channel metal detection sensor uses two different frequencies between antenna sets $\# 1$ and \#2 to avoid interference. In frequency domain, this interference can be minimized by raising the frequency selectivity of receiver. Assume the characteristic of bandpass filter $(\mathrm{BPF})$ in receiver $\# 1$ is $\left|H_{R 1}(f)\right|$ and $\left|H_{R 2}(f)\right|$ for receiver \#2 and power spectral density of incoming signal is $S_{1 \text { In }}(f)$ for receiver $\# 1$ and $S_{2 \text { In }}(f)$ for receiver $\# 2$. Then power spectral densities $S_{1 \mathrm{BPF}}(f)$ of receiver \#1 and $S_{2 \mathrm{BPF}}(f)$ of receiver \#2 become as in

$$
\left[\begin{array}{l}
S_{1 \mathrm{BPF}}(f) \\
S_{2 \mathrm{BPF}}(f)
\end{array}\right]=\left[\begin{array}{ll}
\left|H_{R 1}(f)\right|^{2} & \left|H_{R 1}(f)\right|^{2} \\
\left|H_{R 2}(f)\right|^{2} & \left|H_{R 2}(f)\right|^{2}
\end{array}\right]\left[\begin{array}{l}
S_{1 \text { In }}(f) \\
S_{2 \text { In }}(f)
\end{array}\right] .
$$

As the bandwidth of BPF becomes narrower, the system will be more immune to ambient noise. However this will also 
increase instability in maintaining center frequency of BPF because component values are subject to change along with temperature change. So there exists the optimum $Q$ value of $\mathrm{BPF}$, from the practical point of view, which is needed to be determined by experiment.

3.2. Noise Rejection Using PSD. The input signal after BPF is fed into phase sensitive detector (PSD) to increase selectivity against interfering noise as in Figure 5. In time domain, the output signal from two receiving antennas, which is connected to cancel each other, is fed to receiver as $V_{\text {In }}$. After $\mathrm{BPF}$, it is filtered to $V_{\mathrm{BPF}}$, and the product of $V_{\mathrm{BPF}}$ with $V_{\mathrm{REF}}$ is again filtered through LPF resulting in $V_{\mathrm{LPF}}$. In case the input signal $V_{\text {In }}$ is the mixture of signals from transmitting antennas $\# 1$ and $\# 2$ and noise $n(t)$, then $V_{\text {In }}$ is expressed as in (14) and $V_{\mathrm{OP}}$ as in (15). Hence,

$$
\begin{gathered}
V_{\mathrm{In}}=E_{1} \sin \left(\omega_{1} t+\theta_{1}\right)+E_{2} \sin \left(\omega_{2} t+\theta_{2}\right)+n(t), \\
V_{\mathrm{BPF}}=V_{\mathrm{In}} * h_{\mathrm{BPF}}(t) \\
=\left[E_{1} \sin \left(\omega_{1} t+\theta_{1}\right)+E_{2} \sin \left(\omega_{2} t+\theta_{2}\right)\right. \\
\left.\quad+n(t)+E_{2} \sin \left(\omega_{2} t+\theta_{2}\right)\right] * h_{\mathrm{BPF}}(t), \\
V_{\mathrm{OP}}=V_{\mathrm{BPF}} \times V_{\mathrm{REF}} \\
=E_{\mathrm{REF}} \sin \left(\omega_{1} t+\theta_{1}\right) \\
\quad \times\left[E_{1} \sin \left(\omega_{1} t+\theta_{1}\right) * h_{\mathrm{BPF}}(t)\right. \\
+E_{2} \sin \left(\omega_{2} t+\theta_{2}\right) * h_{\mathrm{BPF}}(t) \\
\left.+n(t) * h_{\mathrm{BPF}}(t)\right] .
\end{gathered}
$$

In case the band-pass filter is ideally tuned to $\omega_{1}$ and lowpass filter ideally cut off unnecessary frequency component, then the output of sensor signal detector $V_{\text {LPF }}$ becomes as in (16). This output signal $V_{\text {LPF }}$ in (16) shows DC signal level which is proportional to receiver input signal with minimum interference:

$$
\begin{aligned}
V_{\mathrm{LPF}}= & V_{\mathrm{OP}} * h_{\mathrm{LPF}}(t) \\
= & E_{\mathrm{REF}} \sin \left(\omega_{1} t+\theta_{1}\right) \\
& \quad \times\left[E_{1} \sin \left(\omega_{1} t+\theta_{1}\right) * h_{\mathrm{BPF}}(t)\right. \\
& \quad+E_{2} \sin \left(\omega_{2} t+\theta_{2}\right) * h_{\mathrm{BPF}}(t) \\
& \left.\quad n(t) * h_{\mathrm{BPF}}(t)\right] * h_{\mathrm{LPF}}(t) \\
\approx & \frac{E_{\mathrm{REF}} E_{1}}{2}+\alpha \cdot n(t) .
\end{aligned}
$$

In case the interfering signal has the same frequency as sensor detection signal, but with different phase relationship, then the signal output after LPF in Figure 5 will be shifted in DC level, depending on the degree of interference, in an ideal case.

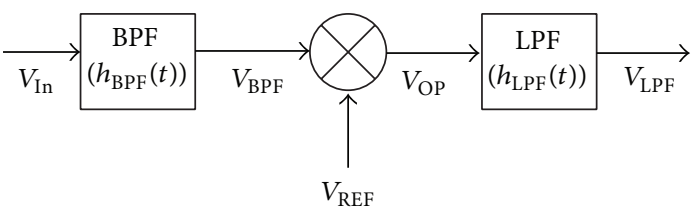

FIGURE 5: Block diagram of sensor signal detector.

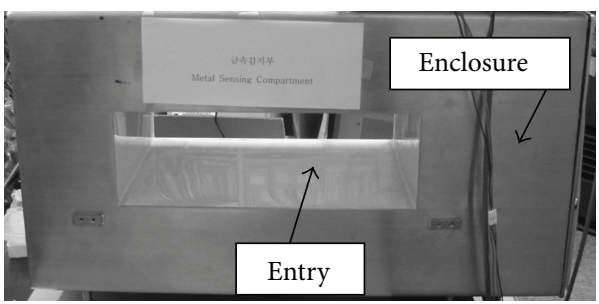

Figure 6: Photo of outer metal enclosure shielding antenna set.

\section{System Design}

4.1. Metal Detection Sensor Having Sensitivity in $\mathrm{mm}$ Scale. Three frequencies, as $50 \mathrm{kHz}, 200 \mathrm{kHz}$, and $400 \mathrm{kHz}$, were designed to be injected into the experimental sensor setup, having sensitivity in $\mathrm{mm}$ scale, to investigate frequency dependant sensitivity while in simultaneous operation with the sensor having sensitivity in $\mathrm{cm}$ scale. Antenna set $\# 1$ was contained in metal enclosure having outer dimension of $\mathrm{W} 55 \times \mathrm{H} 29 \times \mathrm{D} 23 \mathrm{~cm}$ with the opening and $\mathrm{W} 30 \times \mathrm{H} 10 \times$ D23 cm for sensing entry to shield outer interfering noise as was shown in Figure 6. Single turn antennas were used for easy balancing between antennas.

The output voltage from receiving antenna pair, which was connected in opposite polarity, was adjusted to produce nearly zero voltage by using two screws as shown in Figure 7. And the degree of null output signal was measured as CMRR (common mode rejection ratio). After adjusting null position, internal cavity of antenna set \#1 was filled with epoxy to be resistant to outer shock or vibration.

The transmitter block diagram for antenna set \#1 is shown in Figure 8 . The $8 \mathrm{MHz}$ crystal oscillator was used for temperature stability, and fundamental frequency was divided into desired frequencies. Time division switch was facilitated to select single or mixed frequencies. A part of this transmitter signal was fed into phase sensitive detector of receiver as synchronous trigger signal source. Antenna matching circuit was used to match antenna impedance with transmitter impedance.

The receiver block diagram for antenna set \#1 is shown in Figure 9. The input signal from receiving antenna pair was fed into antenna matching circuit not only for impedance matching but also for boosting voltage. After amplification in PRE AMP block and filtering interfering signal in BPF block, the phase of input signal was compared with synchronous trigger signal in PSD block. Finally high frequency component was filtered out in LPF block, and only DC component, proportional to phase difference, appeared and was amplified in AMP block as signal output. The microprocessor was used 


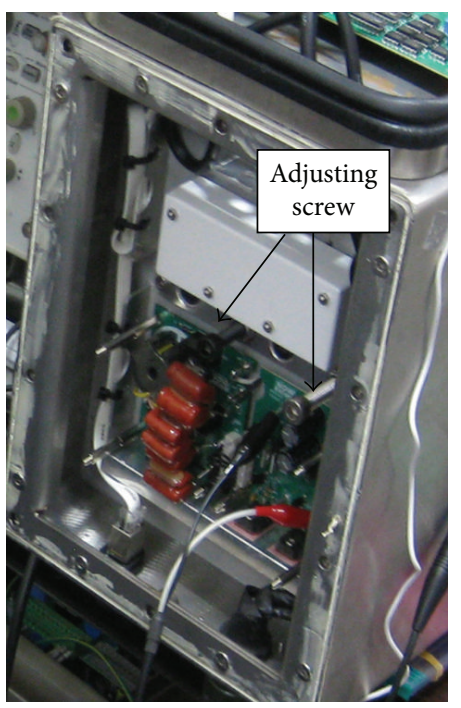

Figure 7: Adjusting screws to produce zero voltage for antenna set $\# 1$.

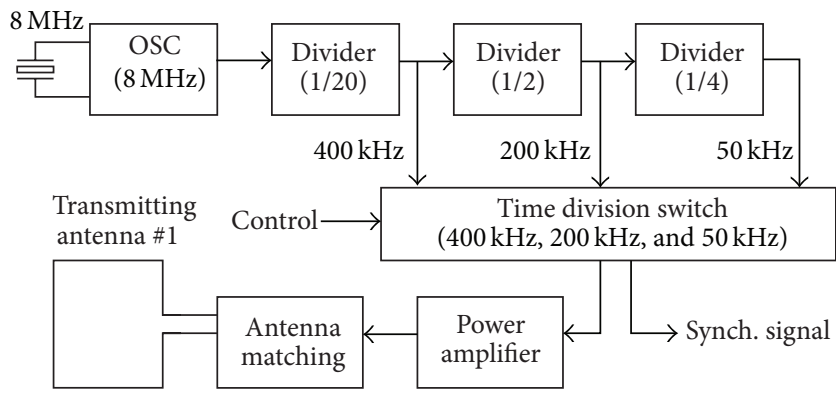

FiguRE 8: Block diagram of transmitter for antenna set \#1.

to control frequency selection and other control parameters in MICOM block.

4.2. Metal Detection Sensor Having Sensitivity in cm Scale. Single $20 \mathrm{kHz}$ frequency was injected into the experimental sensor setup, having sensitivity in $\mathrm{cm}$ scale, to investigate interfering effect with the former sensor, having sensitivity in $\mathrm{mm}$ scale, while in simultaneous operation. The arrangement of antenna set \#2 was devised to be perpendicular with antenna set \#1 to avoid interference. Multiturn antennas were used to compensate sensitivity deficiency due to relatively long distance between transmitting and receiving antennas as shown in Figure 10. Transmitting antenna was fabricated by using $\mathrm{CNC}$ machine to obtain sufficient thickness and reduce resistance, and receiving antennas were fabricated by patterning on PCB. Receiving antenna pair is connected in opposite polarity and adjusted to near zero offset.

The transmitter block diagram is shown in Figure 11. The exciting frequency was adjustable by using potentiometer to select frequency for optimum operation.

In the receiver side as shown in Figure 12, the input signal from receiving antenna pair was directly amplified in PRE AMP block without antenna matching circuit. After

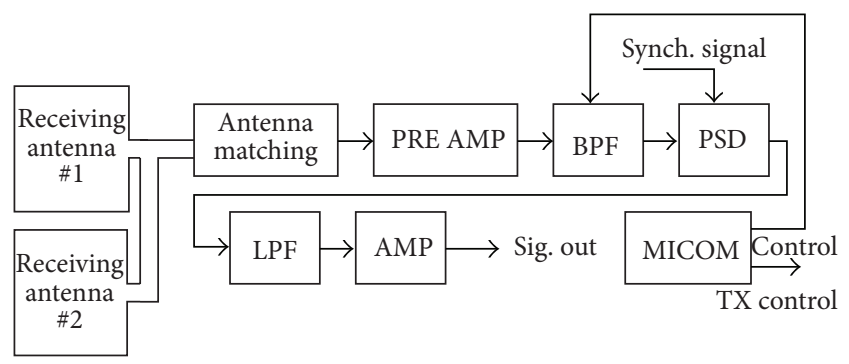

FIGURE 9: Block diagram of receiver for antenna set \#1.

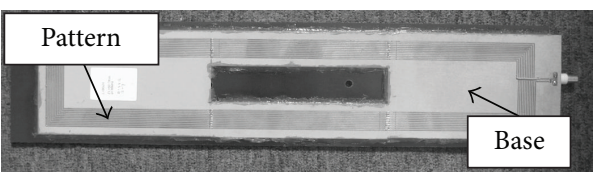

(a)

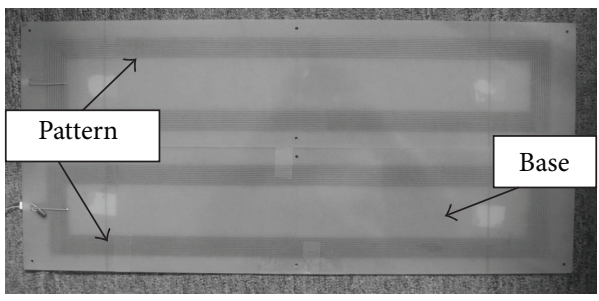

(b)

FIGURE 10: Photo of antenna set \#2: (a) transmitting antenna and (b) receiving antennas.

filtering interference signal in BPF block, the phase of input signal was compared with synchronous trigger signal from transmitter in PSD block. Finally high frequency component was filtered out in LPF block and amplified in AMP block as signal output. Only DC component, proportional to phase difference between input signal and synchronous trigger signal, appeared as signal output.

\section{Measurement}

5.1. Metal Detection Sensor Having Sensitivity in $\mathrm{mm}$ Scale. For this sensing channel, the sensitivity is the key part of the sensor. The minimum size of detectable metal piece is related to the degree of canceling signals from receiving antenna pair, because this limits the maximum amplification ratio. And this figure of merit (FOM) is represented by CMRR, which is the logarithmic value of differential output, $2 \mathrm{mV}_{\mathrm{pp}}$ (before amplification), over single channel output, $10 \mathrm{~V}_{\mathrm{pp}}$. The measured CMRR was $-74 \mathrm{~dB}$ as shown in

$$
\mathrm{CMRR}[\mathrm{dB}]=20 \log \left(\frac{0.2 \mathrm{~V} / \text { gain }}{10 \mathrm{~V}}\right)=-74[\mathrm{~dB}] .
$$

The dependency of output voltage on ferrous metal size was investigated, using ferrous test balls having diameter of $0.8 \mathrm{~mm}, 1.0 \mathrm{~mm}$, and $1.2 \mathrm{~mm}$ at $50 \mathrm{kHz}$ exciting frequency and $33 \mathrm{~Hz}$ LPF (low-pass filter) cutoff frequency after PSD (phase sensitive detector). Measurement data showed that 


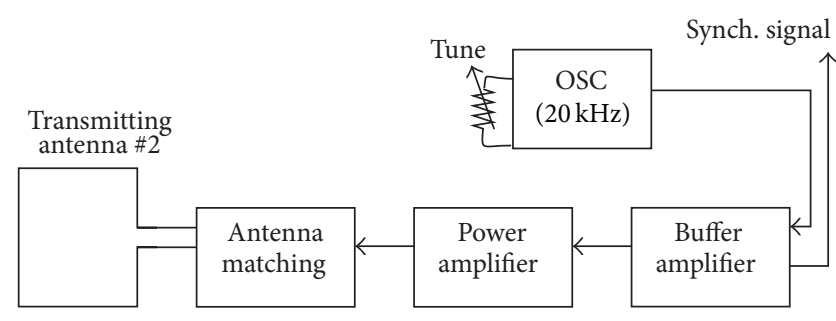

FIGURE 11: Block diagram of transmitter for antenna set \#2.

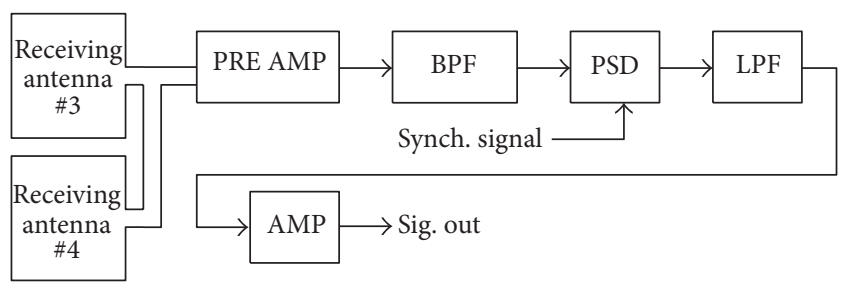

FIGURE 12: Block diagram of receiver for antenna set \#2.

output voltage was almost linearly proportional to the volume as in Figure 13. The minimum detectable size was up to the diameter of $0.8 \mathrm{~mm}$ using $33 \mathrm{~Hz}$ LPF (low-pass filter) cutoff frequency.

The dependency of output voltage on applying frequency $(50 \mathrm{kHz}, 200 \mathrm{kHz}$, and $400 \mathrm{kHz}$ ) was investigated using $\mathrm{Fe}$ ball of $1.2 \mathrm{~mm}$ diameter as shown in Figure 14. Measurement data showed that output voltage was increasing with applying frequency, and this was closely matched if we multiplied the frequency characteristics of antenna matching circuit to the theoretically expected value.

The metal detection sensor using differential loop antennas usually suffers a nonuniform sensitivity distribution inside hollow center area of coil which is used for sample passage due to the nature of loop coil. It is necessary, ideally, to maintain equal sensitivity throughout sensing area. Otherwise the sample metal ball will be undetectable when passing the center area, even if it was detectable when passing edge area. Therefore it is necessary to compensate sensitivity distribution for close to equal sensitivity as possible. The sensitivity distribution was measured for the above sensing entry, W300 $\mathrm{mm} \times \mathrm{H} 100 \mathrm{~mm}$, by applying $50 \mathrm{kHz}$ and using Fe test ball of $1.2 \mathrm{~mm}$ diameter as shown in Figure 15(a). Two small copper plates on center of receiving antenna coil, in horizontal direction, were patched to compensate sensitivity in sensing entry by providing more electromagnetic flux linkages. Measurement data showed that the sensitivity was $-6 \mathrm{~dB}$ at the center of sensing entry as shown in Figure 15(b), which was $+4 \mathrm{~dB}$ enhancement in comparison to the case without patches.

The bandwidth of LPF after PSD is critical for sensitivity enhancement. In the conventional LPF, the narrower the bandwidth is, the lower the noise level is. However, for the metal sensor for moving object detection, the sensitivity is degraded if the bandwidth of LPF is too narrow, because frequency component due to moving object is attenuated. On the contrary, the noise level soars if the bandwidth of LPF is

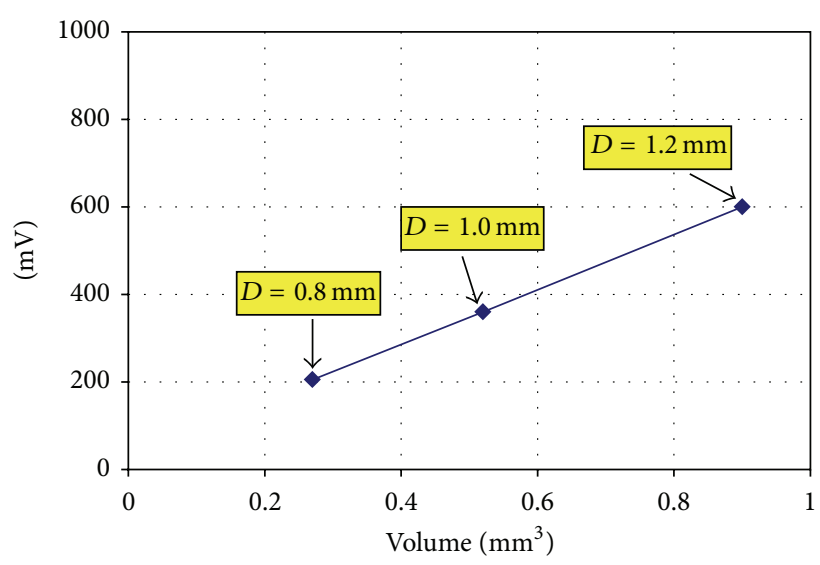

FIGURE 13: Signal output versus Fe ball volume.

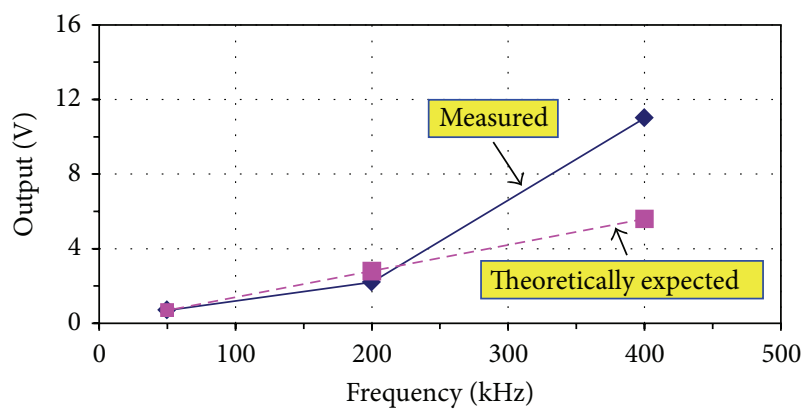

FIGURE 14: Signal output versus applying frequency using Fe ball, $D=1.2 \mathrm{~mm}$.

too wide, resulting in degraded SNR (signal to noise ratio). Also the improved SNR by narrowing the bandwidth of LPF does not mean increasing sensitivity unless it is amplified. Therefore the signal of this ultrahigh sensitivity metal sensor, operating close to detection limit, is only able to be amplified after lowering noise level without sacrificing usable frequency component of moving object. The effect of LPF bandwidth was measured, by varying cutoff frequency, to find optimum sensitivity when exciting frequency of $50 \mathrm{kHz}$ is applied. During the experiment, the signal was amplified for the level which was equivalent to decreasing noise floor, while keeping overall system gain, because there did not exist a margin for signal amplification due to inherent noise level for ultrahigh sensitivity metal sensor operating close to detection limit. The cutoff frequencies of LPF, from $33 \mathrm{~Hz}$ to lower than $11 \mathrm{~Hz}$, were attempted, and the frequency below $11 \mathrm{~Hz}$ resulted in weaker signal output due to too deep attenuation of signal frequency component. The cutoff frequency of $11 \mathrm{~Hz}$ showed the best performance for the moving object which was similar to practical application. The signal responses at $11 \mathrm{~Hz}$ cutoff frequency together with $33 \mathrm{~Hz}$ cutoff frequency are shown in Figure 16 for comparison purpose.

Further test is conducted by applying $50 \mathrm{kHz}$ for ferrous test sample ball having $0.8 \mathrm{~mm}$ and $0.7 \mathrm{~mm}$ diameter using the same $\operatorname{LPF} f_{\text {co }}=11 \mathrm{~Hz}$ to find detection limit as shown in Figure 17. Measurement showed that this metal sensor was able to detect ferrous test sample ball down to $0.7 \mathrm{~mm}$ 


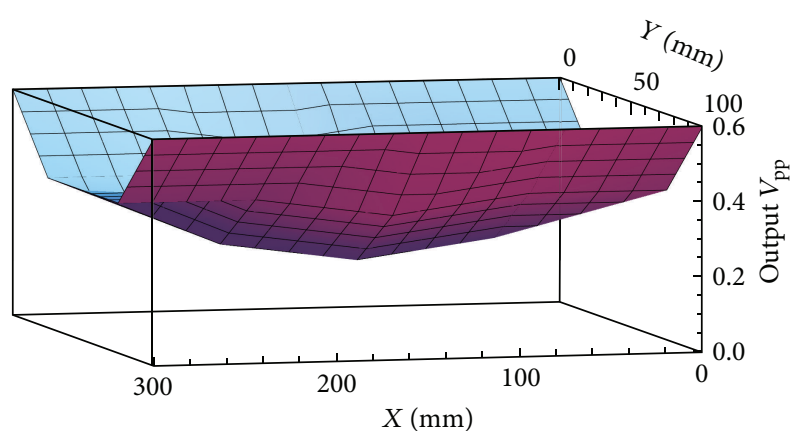

(a)

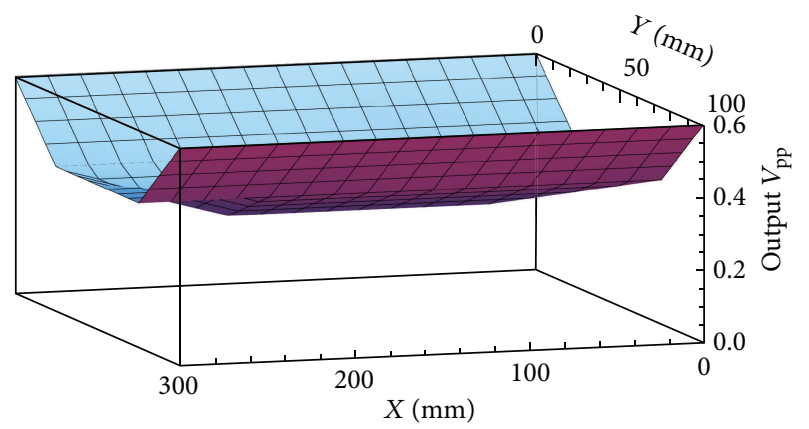

(b)

FIGURE 15: Sensitivity plot of sensing entry (output voltage versus position in sensing entry using Fe $D=1.2 \mathrm{~mm}$ ball): (a) before patching and (b) after patching.

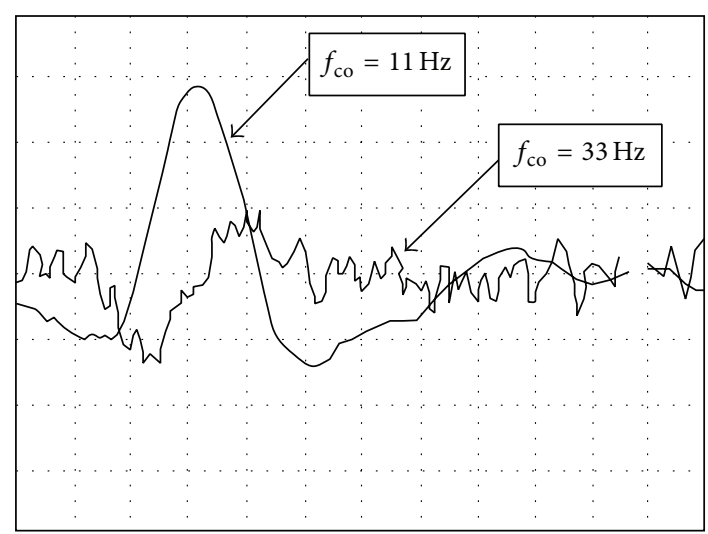

Figure 16: The effect of cutoff frequency of LPF for $D=1.2 \mathrm{~mm}$ ferrous test ball, $Y=200 \mathrm{mV} / \mathrm{div}, X=100 \mathrm{~ms} /$ div.

diameter. Thus the minimum detectable size of Fe ball was extended from $0.8 \mathrm{~mm}$ diameter to $0.7 \mathrm{~mm}$ diameter by optimizing the cutoff frequency of LPF.

5.2. Metal Detection Sensor Having Sensitivity in cm Scale. Measured data showed that the previous ultrahigh sensitivity metal sensor had the linear response range of about $0.7 \mathrm{~mm} \sim$ $4 \mathrm{~mm}$ of Fe ball diameter when the exciting frequency was $50 \mathrm{kHz}$ and cutoff frequency of LPF was $11 \mathrm{~Hz}$. Over this size limit, the sensor detects the presence of metal, but the output response becomes saturated and unable to set further

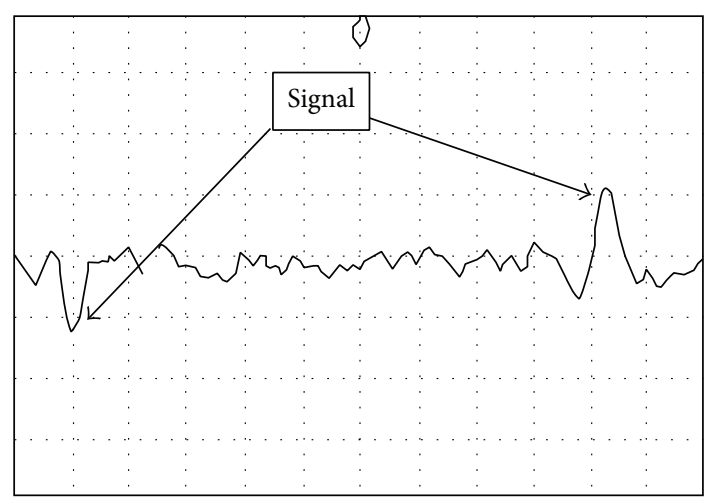

(a)

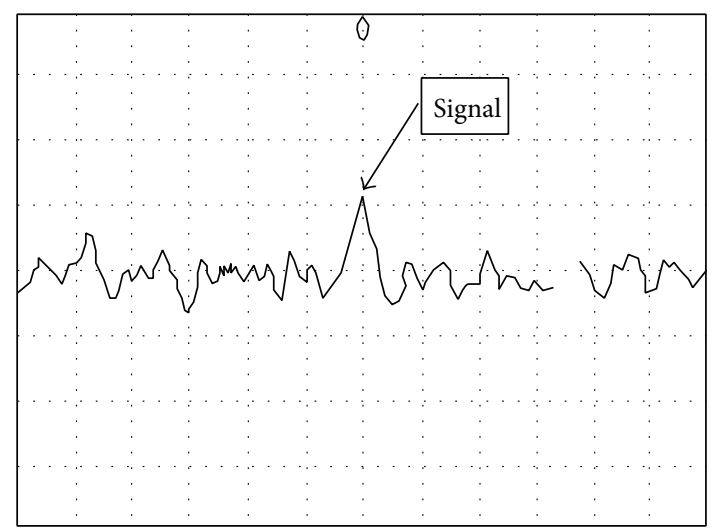

(b)

FIGURE 17: Detection limit of metal sensor: (a) $D=0.8 \mathrm{~mm}$ ferrous test ball $(Y=50 \mathrm{mV} / \mathrm{div}, X=200 \mathrm{~ms} / \mathrm{div})$, (b) $D=0.7 \mathrm{~mm}$ ferrous test ball $(Y=20 \mathrm{mV} / \mathrm{div}, X=200 \mathrm{~ms} / \mathrm{div})$.

threshold point for different size of Fe test ball. Of course, the detectable size can be altered by varying the sensor gain; however the detection range remains similar to the above. The metal sensor having sensitivity in $\mathrm{cm}$ scale was devised with the flexibility to locate the other sensor in adjacent axis to compensate direction dependent sensitivity. Thus this sensor enables sensing throughout wide range of metal sizes from $\mathrm{mm}$ to $\mathrm{cm}$ scale, if it is combined with the previous ultrahigh sensitivity metal sensor.

The single channel response was measured, with reduced gain, to characterize the frequency characteristics of sensor by using TX antenna \#2 and RX antenna \#3, as shown in Figure 18. In the experiment test metal plate occupying 66\% of antenna surface was inserted to find the effect of metal in sensitivity through projected frequency range. The high peak around $50 \mathrm{kHz}$ was observed, and this was regarded as frequency matching characteristics between antenna and transmitter. However the effect of metal piece was similar within the range $40 \sim 60 \%$, which was defined as output voltage after inserting versus output voltage before inserting, as shown in Figure 19. It is noted that the sensitivity is increasing along with the frequency increase as expected, even if the absolute value of output voltage is decreasing in higher frequency region due to frequency matching characteristics. 


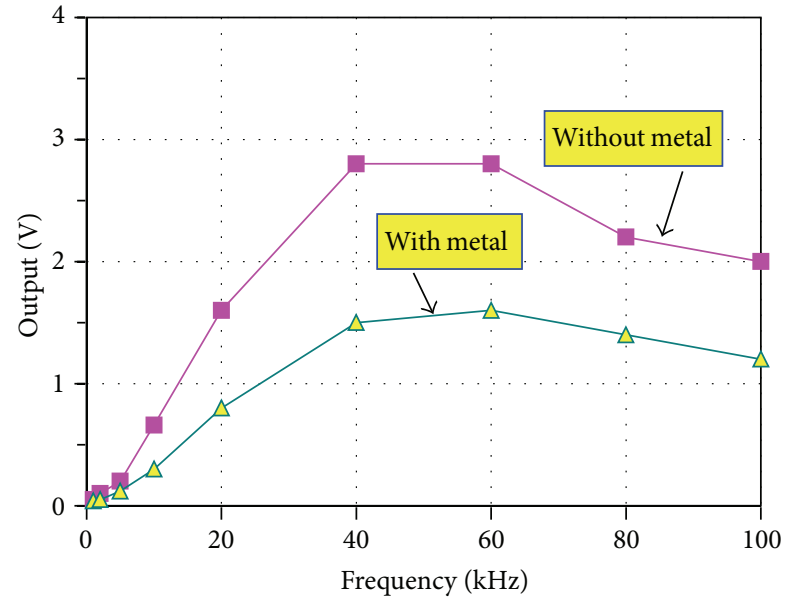

FIGURE 18: Signal output of receiver \#2 for single channel using RX antenna \#3, $X=$ frequency, $Y=$ A.U.

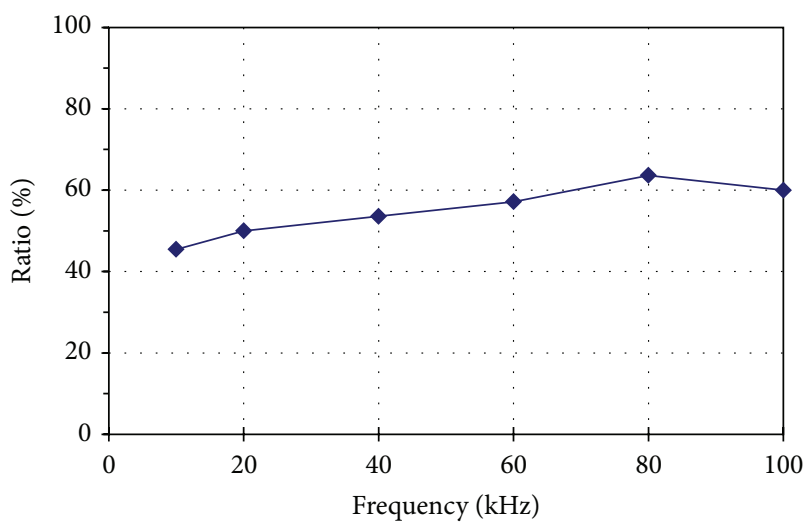

FIGURE 19: Effect of frequency in metal sensing, output voltage after inserting versus before inserting, $X=$ frequency, $Y=$ A.U.

The effect of distance " $d$ " between transmitting antenna and receiving antenna was investigated by separating the distance by two times, and the ratio was calculated, the output voltage at $L=2 d$ over the output voltage at $L=d$, as shown in Figure 20. The measured result shows the tendency, close to the rule of $\propto 1 / d^{2}$. The deviation between the ideal data and measured data is regarded due to leaked electromagnetic fluxes as the distance is farther.

The sensitivity distribution, in horizontal direction, was investigated by gradually covering the antenna along the midline between transmitting antenna and receiving antenna with test metal plate. The measured data showed fairly good horizontal linearity, only $6.5 \%$ nonlinearity. The sensitivity variation, in vertical direction, was measured as $\sim 13 \%$ along the $20 \sim 80 \%$ line connecting between transmitting antenna and receiving antenna. This implies that the loop antenna configuration facing each other provides more uniform distribution in sensitivity.

Following the single channel experiment, two receiving antennas were connected in differential mode, and the gain was increased accordingly. The CMRR was measured in a

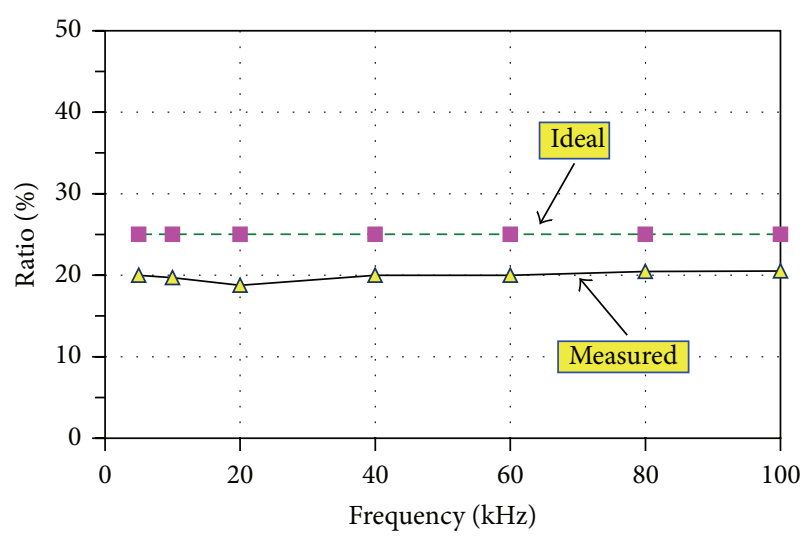

FIGURE 20: Effect of distance between transmitting and receiving antennas, $X=$ frequency, $Y=$ A.U.

TABLE 1: Sensitivity comparison along with test sample laying direction.

\begin{tabular}{|c|c|c|c|}
\hline \multirow[b]{2}{*}{ Sensitivity } & \multicolumn{3}{|c|}{ Direction } \\
\hline & $\begin{array}{c}X \text { direction } \\
(\mathrm{mV})\end{array}$ & $\begin{array}{c}Y \text { direction } \\
(\mathrm{mV})\end{array}$ & $\begin{array}{c}Z \text { direction } \\
(\mathrm{mV})\end{array}$ \\
\hline Peak output voltage (A.U.) & 110 & 80 & 210 \\
\hline
\end{tabular}

similar way to the former sensor channel and was calculated as $-52 \mathrm{~dB}$, less than $22 \mathrm{~dB}$ in comparison to the former sensor. Thus this sensor channel is regarded as adequate for metal object having dimension in $\mathrm{cm}$ scale. Ferrous test sample balls having dimension of $10 \times 5 \times 5(\mathrm{~mm})$ were tested to find the sensitivity in 3 axes. And the result is summarized in Table 1. If we extend the concept of antenna configuration in horizontal direction instead of the present vertical direction, then we can obtain rotated sensitivity distribution. In case we cascade these two antenna configurations, then we are able to obtain more uniform sensitivity distribution by compensating the sensitivity to each other.

5.3. Simultaneous Operation of Two Sensors. The objective of this research is to obtain wide range of sensor responses throughout the object from $\mathrm{mm}$ scale to $\mathrm{cm}$ scale by cascading two sensors having different sensitivity and distribution. However metal detection sensor, operating in differential mode, maintains extremely high gain to increase sensitivity; thus it is strongly influenced by nearby electromagnetic waves. Therefore the effect from the other sensor is mainly investigated through measurement using the present experimental setup to examine the feasibility of this concept. Since the experimental setup was extremely sensitive to outside vibration and electromagnetic waves, all antennas were firmly mounted, adjusted, and fixed using epoxy resin; thus it was not feasible to change parameters such as dimensions and distances. The response from the sensor having sensitivity in $\mathrm{mm}$ scale showed lower response of $\sim 30 \%$ as shown in Figure 21, while in simultaneous operation with the sensor having sensitivity in $\mathrm{cm}$ scale, in comparison to standalone operation. This is considered from the characteristics of PSD because the output from PSD has the tendency of decreasing 


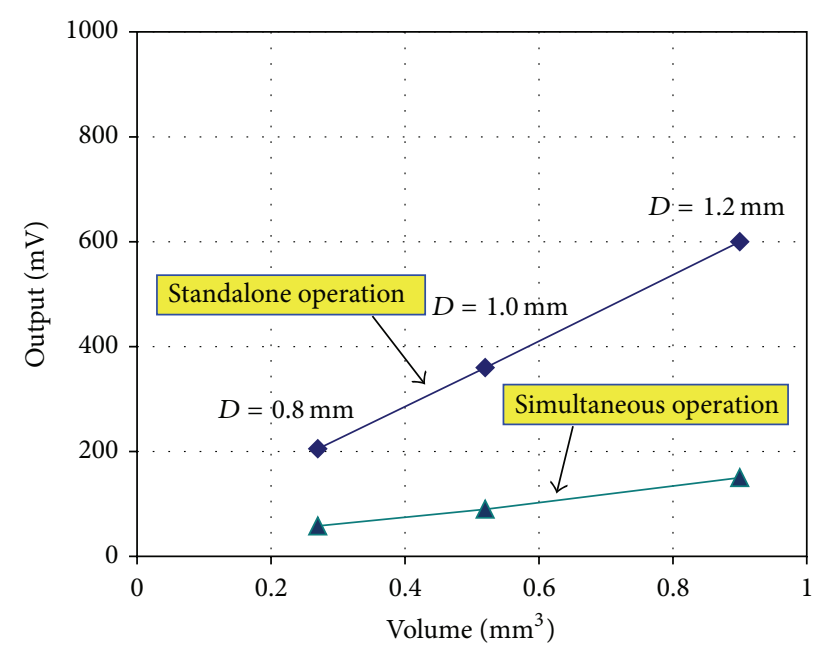

FIGURE 21: Effect of simultaneous operation of two sensors.

along with the increasing outside noise. However it was not possible to decouple each effect individually, such as BPF effect, shielding effect, and PSD effect, due to the delicately integrated sensor system.

On the contrary case, the response from the sensor having sensitivity in $\mathrm{cm}$ scale showed a little less response of $\sim 85 \%$, while in simultaneous operation with the sensor having sensitivity in $\mathrm{mm}$ scale, in comparison to standalone operation. This is considered also from the characteristics of PSD, but with less effect due to lower gain level of the sensor having sensitivity in $\mathrm{cm}$ scale.

\section{Conclusion}

The characteristics of metal detection sensor having two sets of perpendicularly oriented sensor antennas were investigated to extend the sensing range from $\mathrm{mm}$ scale to $\mathrm{cm}$ scale with less interference by cascading two sensors. Metal detection sensor having sensitivity in $\mathrm{mm}$ scale had superior sensitivity to ferrous sphere with diameter down to $0.7 \mathrm{~mm}$ using $50 \mathrm{kHz}$ exciting frequency in standalone operation. Sensor response was proportional to the exciting frequency and the volume of ferrous test sample as expected. The sensitivity distribution at the object passage showed enhanced uniformity by attaching copper patch on the winding coil. The bandwidth, around $11 \mathrm{~Hz}$, of LPF after phase sensitive detector was found to be optimum for sensitivity enhancement. Metal detection sensor having sensitivity in $\mathrm{cm}$ scale showed more uniform sensitivity distribution, but with order of lower sensitivity, which was suited to extend sensing range to $\mathrm{cm}$ scale with minimum interference. This antenna structure, facing each other, has the advantage of adding more axes in a simple way; thus it enables modular construction to achieve near uniform sensitivity distribution without direction dependent sensitivity. The effect of interference while in simultaneous operation of two sensors was investigated, and the measured result showed reduced output response but still within usable detection range. Thus it was feasible to operate two sensors, having different sensitivity range, simultaneously and to extend detection range from $\mathrm{mm}$ to $\mathrm{cm}$ scale, within practically acceptable interference.

\section{Conflict of Interests}

The author declares that there is no conflict of interests regarding the publication of this paper.

\section{Acknowledgment}

This work was supported by Incheon National University Research Grant in 2013.

\section{References}

[1] I. T. McMichael, E. C. Nallon, V. P. Schnee, W. R. Scott, and M. S. Mirotznik, "EBG antenna for GPR colocated with a metal detector for landmine detection," IEEE Geoscience and Remote Sensing Letters, vol. 10, no. 6, pp. 1329-1333, 2013.

[2] M. S. Sharawi and M. I. Sharawi, "Design and implementation of a low cost VLF metal detector with metal-type discrimination capabilities," in Proceedings of the IEEE International Conference on Signal Processing and Communications (ICSPC '07), pp. 480483, November 2007.

[3] T. Miyakawa and K. Honjo, "Development of instrument detecting nonmetal foreign bodies in food material," IEEE Transactions on Instrumentation and Measurement, vol. 43, no. 2, pp. 359-363, 1994.

[4] M. Zourob, S. Mohr, and N. J. Goddard, "Integrated deepprobe optical waveguides for label free bacterial detection," in Proceedings of the International Symposium on Signals, Systems and Electronics (ISSSE '07), pp. 49-52, Montreal, Canada, August 2007.

[5] B. Liu and W. Zhou, "The research of metal detectors using in food industry," in Proceedings of the International Conference on Electronics and Optoelectronics (ICEOE '11), vol. 4, pp. V4-43V4-45, Dalian, China, July 2011.

[6] J. Kwon, J. Lee, and W. Kim, "Real-time detection of foreign objects using x-ray imaging for dry food manufacturing line," in Proceedings of the 12th IEEE International Symposium on Consumer Electronics (ISCE '08), pp. 1-4, April 2008.

[7] W. S. Hua, J. R. Hooks, W. J. Wu, and W. C. Wang, "Development of a polymer based fiberoptic magnetostrictive metal detector system," in Proceedings of the International Symposium on Optomechatronic Technologies (ISOT '10), pp. 1-5, Toronto, Canada, October 2010.

[8] T. Nagaishi, F. Kamitani, H. Ota et al., "First practical high Tc SQUID system for the detection of magnetic contaminants in commercial products," IEEE Transactions on Applied Superconductivity, vol. 17, no. 2, pp. 800-803, 2007.

[9] H. Krause, G. I. Panaitov, N. Wolters et al., "Detection of magnetic contaminations in industrial products using HTS SQUIDs," IEEE Transactions on Applied Superconductivity, vol. 15, no. 2, pp. 729-732, 2005.

[10] S. Yamazaki, H. Nakane, and A. Tanaka, "Basic analysis of a metal detector," in Proceedings of the 18th IEEE Instrumentation and Measurement Technology Conference, vol. 1, pp. 474-477, May 2001. 
[11] S. Yamazaki, H. Nakane, and A. Tanaka, "Basic analysis of a metal detector," IEEE Transactions on Instrumentation and Measurement, vol. 51, no. 4, pp. 810-814, 2002.

[12] P. P. Silvester and D. Omeragic, "Sensitivity of metal detectors to spheroidal targets," IEEE Transactions on Geoscience and Remote Sensing, vol. 33, no. 6, pp. 1331-1335, 1995.

[13] Z. Tang and L. Carter, "Metal detector head analysis," in Proceedings of the 5th International Conference on Sensing Technology, pp. 93-96, Palmerston North, New Zealand, 2011.

[14] K. R. Blay, F. Weiss, D. A. Clark, G. J. J. B. de Groot, M. Bick, and D. Sen, "Signal processing techniques for improved performance of a SQUID-based metal-detector," IEEE Transactions on Applied Superconductivity, vol. 19, no. 3, part 1, pp. 812-815, 2009.

[15] M. Herrmann and K. Sakai, "Objects in powders detected and imaged with $\mathrm{THz}$ radiation," in Proceedings of the IEEE Conference on Lasers and Electro-Optics (CLEO '00), San Francisco, Calif, USA, May 2000.

[16] M. Brighton and M. J. English, "Calculation of optimum spacing for a three coil axially symmetric metal detector," Electronics Letters, vol. 29, no. 10, pp. 838-839, 1993.

[17] P. P. Silvester and D. Omeragić, "Sensitivity maps for metal detector design," IEEE Transactions on Geoscience and Remote Sensing, vol. 34, no. 3, pp. 788-792, 1996. 

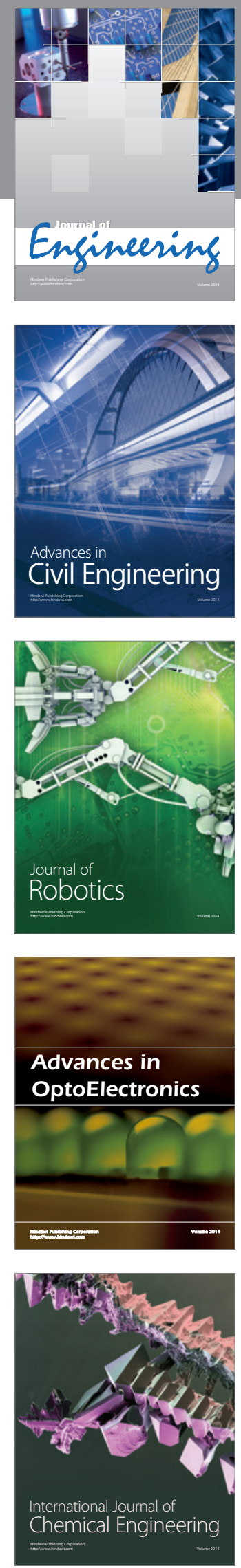

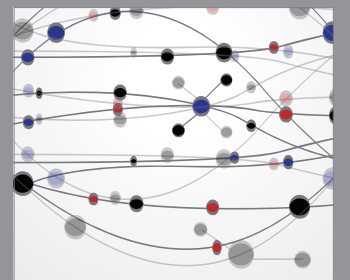

The Scientific World Journal
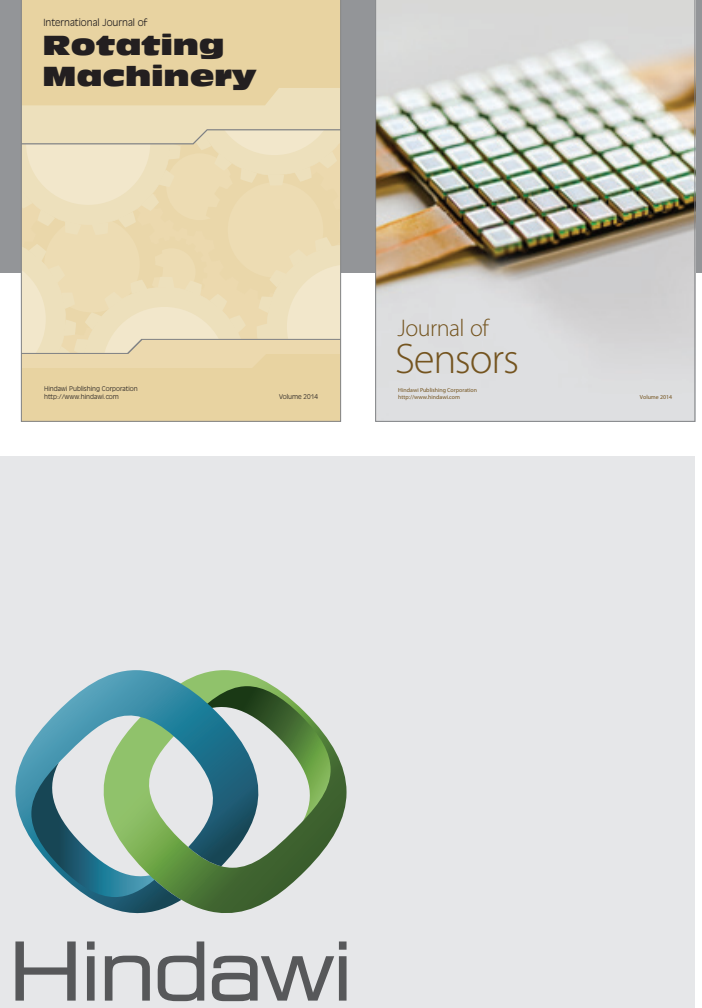

Submit your manuscripts at http://www.hindawi.com
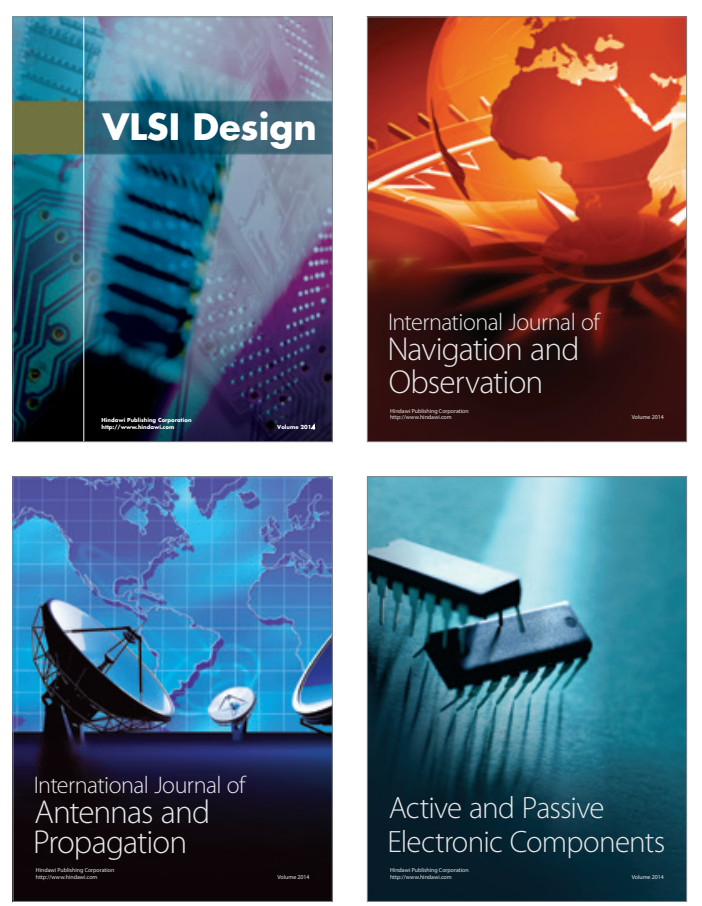
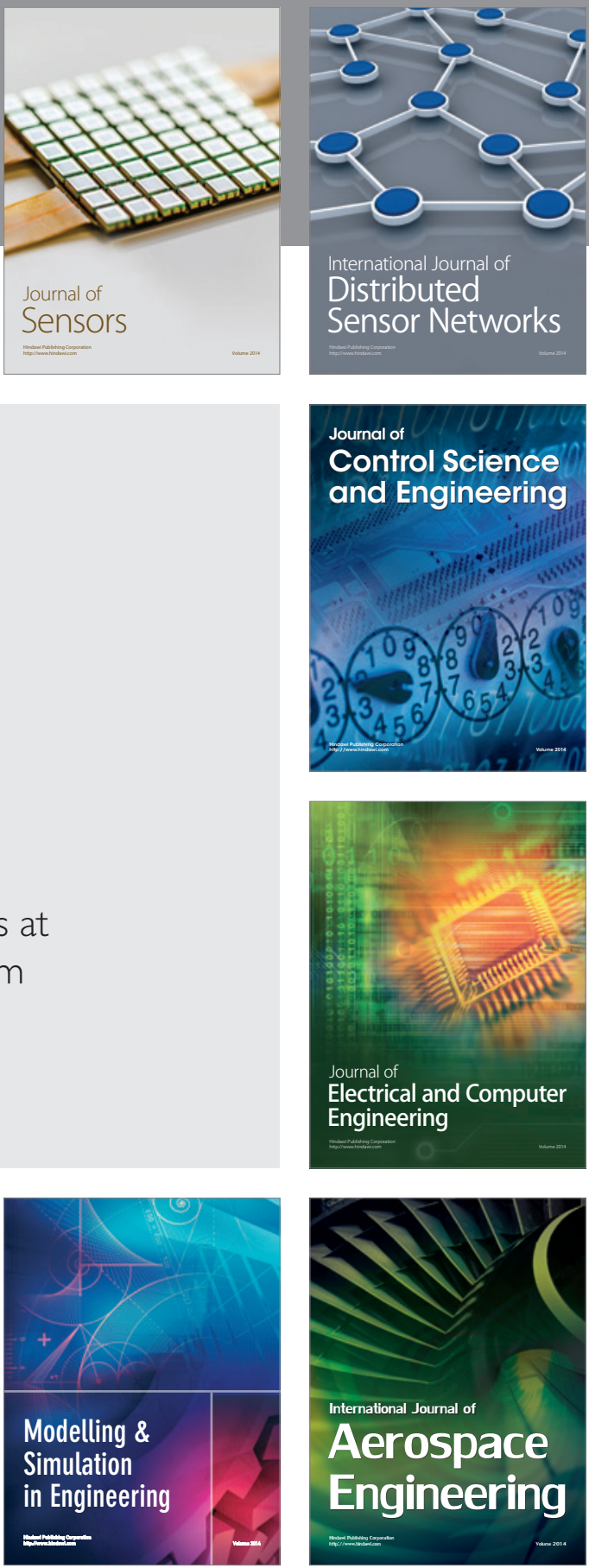

Journal of

Control Science

and Engineering
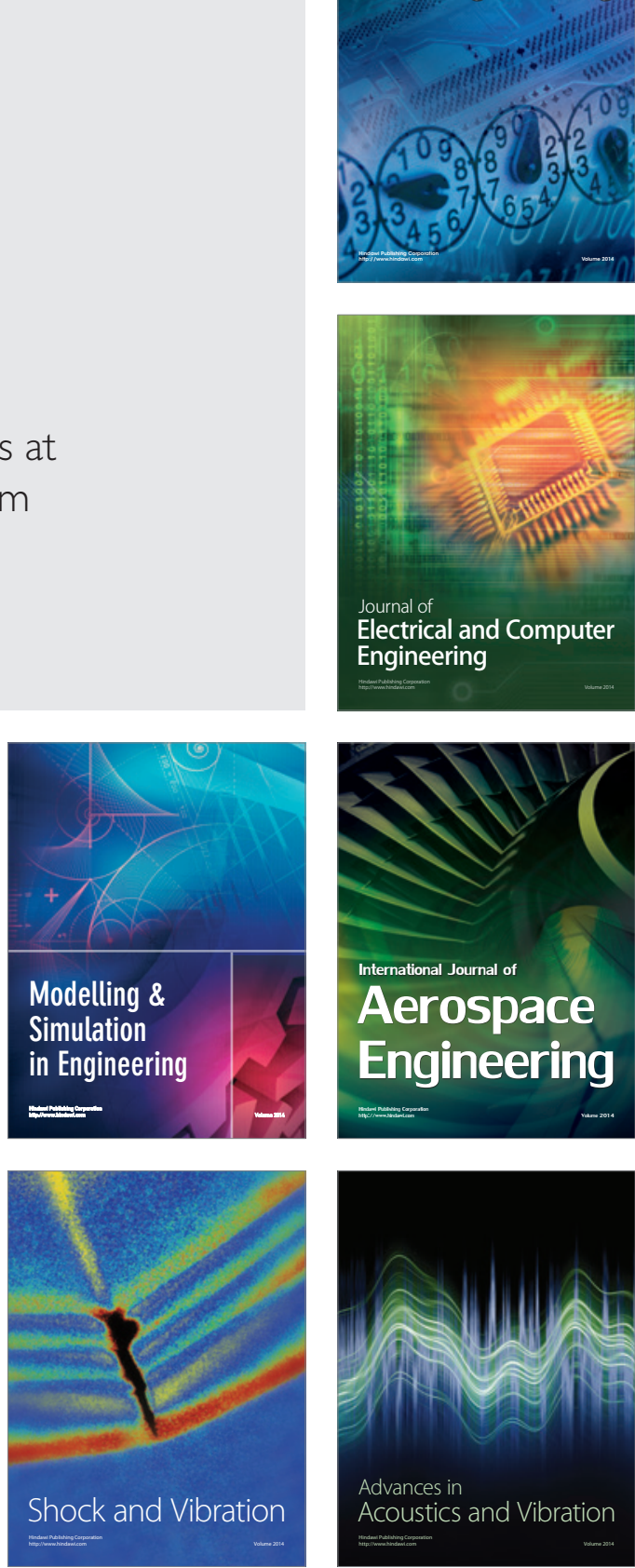